\title{
NEMOTAM: tangent and adjoint models for the ocean modelling platform NEMO
}

\author{
A. Vidard ${ }^{1}$, P.-A. Bouttier ${ }^{2}$, and F. Vigilant ${ }^{1}$ \\ ${ }^{1}$ Inria, Université Grenoble Alpes, CNRS, LJK, 38000 Grenoble, France \\ ${ }^{2}$ Université Grenoble Alpes, CNRS, LGGE, 38000 Grenoble, France \\ Correspondence to: A. Vidard (vidard@imag.fr)
}

Received: 22 September 2014 - Published in Geosci. Model Dev. Discuss.: 10 October 2014

Revised: 6 March 2015 - Accepted: 30 March 2015 - Published: 29 April 2015

\begin{abstract}
Tangent linear and adjoint models (TAMs) are efficient tools to analyse and to control dynamical systems such as NEMO. They can be involved in a large range of applications such as sensitivity analysis, parameter estimation or the computation of characteristic vectors. A TAM is also required by the $4 \mathrm{D}$-Var algorithm, which is one of the major methods in data assimilation. This paper describes the development and the validation of the tangent linear and adjoint model for the NEMO ocean modelling platform (NEMOTAM). The diagnostic tools that are available alongside NEMOTAM are detailed and discussed, and several applications are also presented.
\end{abstract}

\section{Introduction and history}

Tangent linear and adjoint models (TAMs in the following) are powerful modelling tools. The tangent linear model (TLM) provides the directional derivatives with respect to a trajectory of the corresponding non-linear system. The adjoint of the TLM gives information about the response of the system to variations of its input. TAMs are therefore widely used for variational assimilation applications as well as for the analysis of physical processes, since they can be used for sensitivity analysis, parameter identification and for the computation of characteristic vectors (singular vectors, Lyapunov vectors, etc.) (see Moore et al., 2004, for an extended review).

This is particularly true for geophysical applications such as meteorology and oceanography where many data assimilation systems rely on the availability of such models. However, only few ocean general circulation models are rou- tinely provided with their TAM. Among them, one can cite MITgcm (MIT general circulation model) and ROMS (Regional Ocean Modelling System). They are quite representative of the two possible routes for deriving tangent and adjoint model, either using automatic differentiation tools (MITgcm uses automatic differentiation; Marotzke et al., 1999) or hand-coded (ROMS' TAM was developed by a pool of researchers; Moore et al., 2004).

Automatic differentiation tools have been available for some time now, and they show some significant advantages over the hand-coded route. Differentiation of a numerical code is a tedious and error-prone task, the use of an automatic tool could alleviate this difficulty. Moreover when an updated version of the non-linear model is provided the corresponding TAM can in general be obtained effortlessly. Additionally automatic tools offer an important flexibility when one wants to change the variable to differentiate around. Indeed, typically, for data assimilation purposes, the TAM is differentiated around the initial condition, while for parameter estimation it is differentiated around the sought parameter set. Most of the time the obtained TAMs are very similar, but in some cases (e.g. for grid-related parameters) going from one to another can lead to a significant number of code changes.

On the other hand, automatic differentiation suffers from some limitations compared to hand-coding. Firstly, some newer (or archaic) language features may not be supported at first by the automatic tools; secondly, the numerical performance of automatically derived TAM is still relatively poor compared to that of the hand-coded one. In particular, the handling of the parallelisation is still an open issue. Moreover, they are not fully automatic since non-differentiable parts of the original code are still required to be dealt with 
by specialists in the field. All these issues can however be overcome by mixing automatic and manual coding.

The NEMO ocean engine (Madec, 2008) was previously known as the OPA model (Madec et al., 1998). It used to have a TAM (called OPATAM), fully hand-coded. OPATAM was initially developed for a Pacific Ocean configuration and targeted variational data assimilation applications in the framework of OPAVAR (Weaver et al., 2003, 2005). OPATAM and OPAVAR were extended to other regional basins (Mediterranean Sea, Rémy, 1999; North Atlantic $1 / 3^{\circ}$, Forget et al., 2008; South Atlantic $1^{\circ}$ ) and to the global ocean (ORCA $2^{\circ}$, Daget et al., 2009). They were used for methodological studies such as control of the 3-D model error (Vidard, 2001), control of the surface forcing and open boundary conditions (Deltel, 2002; Vossepoel et al., 2003). OPATAM was also used for sensitivity studies (Sévellec et al., 2008) and singular vectors (Moore et al., 2003; Sévellec et al., 2009).

For several reasons, mainly because of lack of workforce, OPATAM, OPAVAR and related developments were not included in the standard release of OPA. As a consequence, synchronisation of OPATAM with OPA's releases could not be achieved on a regular basis, and all developments were on individual branches, without feedback to the OPATAMOPAVAR system. The pool of potential users was therefore reduced significantly. The complete rewriting of the model during the transition to NEMO rendered OPATAM and OPAVAR obsolete. As part of the NEMOVAR initiative (a variational data assimilation with NEMO, Mogensen et al., 2009) a first prototype of NEMOTAM was obtained by Tber et al. (2007) using the TAPENADE automatic differentiation tool (Hascoët and Pascual, 2004) for a fixed and somewhat simplified configuration (ORCA $2^{\circ}$ with all nondifferentiable options switched off, Tber et al., 2007). This initiative was successful in a reasonable amount of time; however, even for this simplified configuration substantial human intervention and additional work was required to obtain an efficient product from the raw generated code. Three main drawbacks were identified for this application. First, the memory management and CPU performance of the raw code were rather poor. Second, the version of TAPENADE at that time generated single-processor code only and could not handle directives from the C-PreProcessor (CPP keys), which are widespread in NEMO. Improved memory management and extensions to support massive parallel processing and CPP keys are planned in future versions of TAPENADE. Even as it is now it can be overcome manually with a reasonable additional effort, so these first two deficiencies are not fundamental. There is a third problem that is an incompatibility between the way automatic differentiation tools handle non-linearities and the so-called incremental 4D-Var assimilation algorithm used in NEMOVAR, which was the original motivation for developing NEMOTAM. Indeed when a nonlinearity occurs in the direct model, the value of the corresponding variable needs to be made available to the tangent and adjoint model to differentiate around. For this purpose, on the one hand automatic differentiation tools run the direct model alongside the tangent and store one way or another the relevant value for the backward integration of the adjoint (e.g. binomial checkpointing as in Tber et al., 2007). On the other hand, incremental 4D-Var would perform a minimisation using only tangent and adjoint integrations, meaning it would run several instances of the tangent model for one instance of the direct (and possibly at a different resolution), so direct and tangent models cannot be run alongside each other.

The modifications required to make any automatic differentiation tool compatible with the multi-incremental approach are really substantial and cannot be done in a short or medium term. Moreover the numerical performances of the automatically generated TAMs do not yet allow its use for "big" configurations and for operational applications. The writing of an adjoint code is not a simple technical task; this explains why automatic differentiation tools can seldom be used as a black box. Numerous potential problems can arise, and in general they should be dealt with on a case-by-case basis by someone who masters both the model and differentiation techniques.

From that experience it has been decided to go forward with the hand-coding approach - the use of automatic differentiation tool being left aside for the time being. We may reconsider this in the medium or long term though. An optimal mix of both approaches is likely to be the preferred choice and lead to a semi-automatic way of generating NEMOTAM.

This paper will first discuss the methodology used for the NEMOTAM development and explain some of the particular choices that have been made. Then the validation tools that are available alongside NEMOTAM will be detailed. Finally some application examples will be presented.

\section{Methodology and choices for NEMOTAM}

The approach used in developing NEMOTAM is based on the well-known differentiation rules as described in Giering and Kaminski (1998). It relies in particular on the definition of active and passive variables. The former depends on the control variables (variables one differentiates around), while the latter are independent of the control (typically model or grid parameters). Active variables have tangent and adjoint counterparts while passive variables do not.

In the current version (3.4.1), only the general circulation component of NEMO is supported by NEMOTAM and a few key components are still missing, namely the variable volume, open boundary conditions and the grid nesting capabilities. There is no fundamental reason for not supporting these options (support for open boundary conditions is actually in progress), but it was not flagged as priority. In particular the "variable volume" option would require a tremendous amount of coding since it makes the surface grid cells size depending on the flow (thus becoming active variables). This 
is a typical case where a semi-automatic route would be beneficial.

When coding tangent and adjoint models, either automatically or manually, one has to face several difficulties. The main ones are listed in the remainder of this section and discussed in the particular case of NEMOTAM.

\subsection{Non-differentiabilities: problem and solutions}

In realistic models like those included in NEMO, nondifferentiable physics are quite frequent. They can be nondifferentiable in essence (e.g. step processes), due to the way they have been programmed or the chosen numerical methods (e.g. non-oscillatory schemes). Coding-wise they can be represented by an IF statement with a condition on an active variable (or a max, abs, etc. statement but it is equivalent). In order to manage these non-differentiable parts, several options are available.

- Regularisation: either mathematical regularisation (thanks to the introduction of a differentiable connection) or a physical regularisation whenever it is possible (by rewriting the processes in a differentiable manner).

- Approximation: the direct model physics is approximated in TAM in order to transform the nondifferentiable part. One should obviously be careful that the approximation is not too strong.

- Non-linear branching: the non-differentiable part is kept in TAM, but the same branching (same side of the IF) as in the direct model is used (i.e. one left differentiates and one right differentiates separately). This is in general the preferred choice, but in some cases it can lead to pathological behaviour and should be done very carefully.

The choice of treatment heavily relies on the type of the non-differentiability, and it is more a matter of educated choice. Indeed it requires important knowledge of both the direct model and differentiation techniques. A typical example of this in NEMO is the vertical mixing schemes included in OPA. The current version is strongly non-differentiable. In NEMOTAM differentiation is achieved by first simplifying the physics: some active variables are computed by the direct model and treated as a passive variable in the TAM. Adopting this strategy has the advantage of keeping the full physics in the non-linear model, but it is at the expense of an approximation in the TAM. However, most of the physical process it models being quite regular, it may be possible to rewrite the direct version to make it differentiable ${ }^{1}$.

Another important source of non-differentiability is the non-oscillatory part of one of the most popular tracer advection schemes in NEMO. This kind of scheme is highly non-

\footnotetext{
${ }^{1}$ This was the strategy adopted in ROMS for the vertical mixing scheme.
}

differentiable, and classical non-linear branching (as an automatic differentiation tool would do) can lead to a very unstable TAM. Following Thuburn (2001) two viable approximations are at hand: either one differentiates at continuous level and then applies the same non-oscillatory discretisation scheme as for the direct model, or the non-oscillatory correcting term is removed altogether in the tangent and adjoint schemes. Both ways present some approximations: the former is generally a better approximation for the tangent model in the long run, but it introduces some non-linearities and degrades the exactness of the adjoint model with respect to the tangent model. For this reason, in NEMOTAM the second solution has been adopted.

\subsection{Checkpointing}

One of the issues one has to tackle when dealing with TAM is the storage and/or re-computation of the non-linear trajectory that is required for the differentiation of the non-linear terms. This is particularly important for the adjoint model, which needs the trajectory in reverse order as it is produced by the non-linear model. A common practice is the so-called checkpointing where snapshots of the trajectory (the checkpoints) are stored and intermediate variables are re-computed between checkpoints. A similar strategy is used in NEMOTAM: a subset of direct variables are stored on disk at the end of every time step, and only required intermediate variables are recomputed. The number of non-linearities in NEMO being relatively small, the need for storage and re-computation is limited. However, for long integrations the amount of required storage may become too severe; in that case a possible approximation is to subsample the output of the trajectory (say one per day), and NEMOTAM will interpolate linearly between checkpoints. Additionally, it can be stored in single precision. The validity of these approximations is discussed in Sect. 3. Automatic differentiation tools generally allow for the use of more efficient multi-level checkpointing, but it is not possible here due to the fact that NEMO and NEMOTAM are not run together.

\subsection{Numerical issues}

The numerical characteristics of the direct model are not always conserved in the adjoint model. In particular the most common difficulty is related to the convergence speed of an iterative algorithm that may be different for the direct and its tangent/adjoint counterparts.

In that case, a specific solution should be provided; it can go as far as replacing the problematic scheme by a more TAM-friendly one. Once again, in OPATAM this kind of problem arose, and one had to replace the conjugate gradient solver used for the computation of the surface pressure gradient (while self-adjoint, in theory) by a red-black successive over-relaxation solver. However, this scheme being on 
the verge of depreciation in NEMO, NEMOTAM will soon adopt the time-splitting-based direct solver.

Stability is not the only numerical issue: the TAMs are, in essence, more expensive numerically than the direct model because of the additional computations they require. Moreover, and it is especially true for the adjoint model, direct code optimisations may not be so optimal for the TAM. Therefore it is important to specifically optimise the TAM code, and this can only be done through a careful performance analysis. As of today, such an optimisation may not be achieved automatically. The tendency toward application at high and very high resolution makes the computing cost aspect a crucial issue. NEMOTAM adopted the same domain decomposition strategy as for NEMO and is therefore fully parallel, and some specific targeted optimisations have been performed. Additionally some irrelevant (as far as TAMs are concerned) on-line diagnostics have been removed. Thanks to all these efforts the additional computing burden has been contained to around twice the non-linear integration cost (more or less, depending on the application).

\section{Validation}

Due to the numerous caveats mentioned in Sect. 2, validation of TAM is a crucial aspect of the model development. Fortunately there are several numerical tests that can be performed to ensure this validity. Moreover, these tests can be applied both to the whole model and to individual modules separately.

In NEMOTAM, modules include both the tangent and adjoint parts, as well as the validation interface, for numerical verification of the TAM source codes. The adjoint test would always be present, while the tangent test could be optional and reserved for specific and problematic routines.

In the following $\mathcal{M}(\boldsymbol{x})$ stands for the full nonlinear NEMO model with initial state vector $\boldsymbol{x}, \mathbf{L}(\boldsymbol{x}) \equiv$ $\left.(\partial \mathcal{M} / \partial \boldsymbol{x})\right|_{\boldsymbol{x}=\boldsymbol{x}}$ its tangent linear model (possibly simplified), and $\mathbf{L}^{*}(\boldsymbol{x})$ the adjoint of $\mathbf{L}(\boldsymbol{x})$.

\subsection{Adjoint validation}

The adjoint part is actually relatively easy to check. Indeed, by definition of the adjoint one obtains

$$
(\mathbf{L}(\boldsymbol{x}) \delta \boldsymbol{x}, \delta \boldsymbol{y})=\left\langle\delta \boldsymbol{x}, \mathbf{L}^{*}(\boldsymbol{x}) \delta \boldsymbol{y}\right\rangle,
$$

where $\langle.,$.$\rangle and (.,$.$) denote the appropriate dot product.$ Equation (1) being exact, the relative error between the two computed scalar products must be close to zero barring rounding errors. In NEMOTAM, the actual test performed is

$$
(\mathbf{L}(\boldsymbol{x}) \delta \boldsymbol{x})^{*} \mathbf{W} \delta \boldsymbol{y}=\delta \boldsymbol{x}^{*} \mathbf{L}^{*}(\boldsymbol{x}) \mathbf{W} \delta \boldsymbol{y},
$$

where $\delta \boldsymbol{x}$ is a random vector, $\mathbf{W}$ is a diagonal matrix of scale factors and $\delta \boldsymbol{y}=\mathbf{L}(\boldsymbol{x}) \delta \boldsymbol{x}$. This test only ensures that the ad- joint is indeed the adjoint of the tangent linear, which in turn has to be validated.

\subsection{Tangent validation}

Validation of tangent modules is more tricky since there are generally no affordable exact tests available. A classical method of testing a numerical tangent linear model $\mathbf{L}$ is to compare the evolution of a perturbation by $\mathbf{L}$ with the difference of two evolutions, with and without the perturbation, by the full non-linear model $\mathcal{M}$.

Considering a fixed small perturbation vector $\delta \boldsymbol{x}_{0}$, and $\gamma$ a scale parameter, the Taylor expansion of $\mathcal{M}$ reads

$\mathcal{M}\left(\boldsymbol{x}_{0}+\gamma \delta \boldsymbol{x}_{0}, t\right)=\mathcal{M}\left(\boldsymbol{x}_{0}, t\right)+\gamma \mathbf{L}(\boldsymbol{x}, t) \delta \boldsymbol{x}_{0}+O\left(\gamma^{2}\right)$.

If $\mathcal{N}\left(\gamma \delta \boldsymbol{x}_{0}, t\right)$ denotes the non-linear evolution of a perturbation,

$\mathcal{N}\left(\gamma \delta \boldsymbol{x}_{0}, t\right)=\mathcal{M}\left(\boldsymbol{x}_{0}+\gamma \delta \boldsymbol{x}_{0}, t\right)-\mathcal{M}\left(\boldsymbol{x}_{0}, t\right)$.

The linearisation error $\mathcal{E}\left(\gamma \delta \boldsymbol{x}_{0}, t\right)$ is defined by

$$
\mathcal{E}\left(\gamma \delta \boldsymbol{x}_{0}, t\right)=\mathcal{N}\left(\gamma \delta \boldsymbol{x}_{0}, t\right)-\gamma \mathbf{L}(\boldsymbol{x}, t) \delta \boldsymbol{x}_{0}
$$

From Eq. (3), $\mathcal{E}\left(\gamma \delta \boldsymbol{x}_{0}, t\right)$ behaves like $O\left(\gamma^{2}\right)$.

The first-order accuracy index $\epsilon_{\gamma}$ is given by

$\epsilon_{\gamma}=\frac{\left\|\mathcal{N}\left(\gamma \delta \boldsymbol{x}_{0}, t\right)\right\|}{\left\|\mathbf{L}(\boldsymbol{x}, t) \gamma \delta \boldsymbol{x}_{0}\right\|}$.

$\epsilon_{\gamma}$ tends to 1 as $\gamma$ tends to 0 , validating $\mathbf{L}(\boldsymbol{x}, t)$. Moreover when $\gamma$ is small enough, $\mathcal{N}$ enters a linear regime and $\epsilon_{\gamma}$ converges toward 1 with a rate $\gamma$.

Table 1 shows an example of such tests on a single routine. It illustrates nicely the expected behaviour of $\epsilon_{\gamma}$, which gains one digit in precision when $\gamma$ is divided by 10 . This diagnostic gives information of both first order (tends to 1) and second order (at rate $\gamma$ ).

\subsection{Estimation of the approximation error}

As mentioned above, when differentiating realistic models, approximations have to be made. To estimate the effect of these approximations on the numerical tangent linear model $\mathbf{L}$, one must first estimate the truncated part of the Taylor expansion of Eq. (3). In order to do this, following Lawless et al. (2003), one can write the Taylor expansion of $\mathcal{E}(\delta \boldsymbol{x}, t)$ whose individual components $l$ follow

$\mathcal{E}_{l}\left(\delta \boldsymbol{x}_{0}, t\right)=\frac{1}{2} \partial^{2} \mathcal{M}_{l} \delta \boldsymbol{x}_{0}^{2}+\frac{1}{6} \partial^{3} \mathcal{M}_{l} \delta \boldsymbol{x}_{0}^{3}+\ldots$

On the other hand, from two non-linear perturbations,

$$
\begin{aligned}
& \mathcal{N}\left(\delta \boldsymbol{x}_{0}, t\right)=\mathcal{M}\left(\boldsymbol{x}_{0}+\delta \boldsymbol{x}_{0}, t\right)-\mathcal{M}\left(\boldsymbol{x}_{0}\right) \\
& \mathcal{N}\left(\gamma \delta \boldsymbol{x}_{0}, t\right)=\mathcal{M}\left(\boldsymbol{x}_{0}+\gamma \delta \boldsymbol{x}_{0}, t\right)-\mathcal{M}\left(\boldsymbol{x}_{0}\right)
\end{aligned}
$$


Table 1. Tangent validity tests for the bn2 routine (computation of the Brunt-Väisälä frequency), values of the first-order accuracy in$\operatorname{dex} \epsilon_{\gamma}$ for several values of the perturbation amplitude $\gamma$.

\begin{tabular}{lcr}
\hline Routine $(\mathbf{L})$ & $\gamma$ & $\epsilon_{\gamma}$ \\
\hline bn2_tan & $1 \times 10^{0}$ & 0.999961862090 \\
bn2_tan & $1 \times 10^{-1}$ & 0.999995878199 \\
bn2_tan & $1 \times 10^{-2}$ & 0.999999584740 \\
bn2_tan & $1 \times 10^{-3}$ & 0.999999958442 \\
bn2_tan & $1 \times 10^{-4}$ & 0.999999995846
\end{tabular}

one can compute

$$
E\left(\gamma \delta \boldsymbol{x}_{0}, t\right)=\frac{\mathcal{N}\left(\gamma \delta \boldsymbol{x}_{0}, t\right)-\gamma \mathcal{N}\left(\delta \boldsymbol{x}_{0}, t\right)}{\gamma^{2}-\gamma},
$$

whose Taylor expansion reads (for each individual component $l$ )

$E_{l}\left(\gamma \delta \boldsymbol{x}_{0}, t\right)=\frac{1}{2} \partial^{2} \mathcal{M}_{l} \delta \boldsymbol{x}_{0}^{2}+\frac{1+\gamma}{6} \partial^{3} \mathcal{M}_{l} \delta \boldsymbol{x}_{0}^{3}+O\left(\gamma^{4}\right)$

For small values of $\gamma$ and $\delta \boldsymbol{x}_{0}$, one can compare $E$ and $\mathcal{E}$. That way one builds up an estimator of the numerical tangent linear model error:

$$
\hat{\mathcal{E}}=100\left(1-\frac{\|E\|}{\|\mathcal{E}\|}\right) \text {. }
$$

Moreover, in NEMO, the vast majority of the non-linearities are quadratic ones, meaning the third order and above derivatives vanish from the Taylor expansion and one gets $E=\mathcal{E}$.

This diagnostic is very valuable when comparing different simplifications made to the tangent linear model. Table 2 shows $\hat{\mathcal{E}}$ for two configurations of NEMO: an academic test case that is fully differentiable (SEABASS; see Appendix A) and $2^{\circ}$-resolution global realistic configuration (ORCA2). This allows us to measure the effect of approximations mentioned in Sects. 2.2 and 2.1

\section{Application examples}

As stated by Errico (1997): "the principal application of adjoint models is sensitivity analysis, and all its other applications may be considered as derived from it". Performing sensitivity analysis means evaluating how variations on the input of the system will affect the output. This can be of use for understanding the behaviour of the system (sensitivity analysis, propagation of uncertainty), for optimising it (through data assimilation for instance) and for performing stability analysis. This section presents an example of these three kinds of applications.
Table 2. Approximation error in the tangent linear model for different configurations over 10 days.

\begin{tabular}{lr}
\hline Configurations & $\hat{\mathcal{E}}$ \\
\hline SEABASS no simplification & negligible \\
SEABASS interpolated checkpoint (1 per day) & negligible \\
SEABASS simplified TVD & $3 \%$ \\
ORCA2 simplified vertical mixing & $1 \%$ \\
ORCA2 idem + TVD + interp. checkpoint (1 per day) & $4 \%$ \\
\hline
\end{tabular}

\subsection{Sensitivity analysis and data assimilation}

In the variational context, sensitivity analysis is the computation of the gradient of so-called response (or cost or objective or criterion) functions with respect to given control vectors. In other words, given the model's state $\boldsymbol{x}=\left(x_{1}, \ldots, x_{n}\right)^{T} \in$ $\mathcal{X} \subset \mathbb{R}^{n}$ and a set of control parameters $\alpha=\left(\alpha_{1}, \ldots, \alpha_{p}\right)^{T} \in$ $\mathcal{P} \subset \mathbb{R}^{p}$, one is interested in computing gradients with respect to $\alpha$ of a given response function:

$$
\begin{aligned}
J: \mathcal{P} & \rightarrow \mathbb{R} \\
\alpha & \longmapsto J(\alpha)=\int_{0}^{T}\|\phi(\boldsymbol{x}, \alpha, t)\|^{2} \mathrm{~d} t,
\end{aligned}
$$

where $\phi$ is a (possibly non-linear) function with values in $\mathbb{R}^{m}$. We considered here the mono-criterion case ( $J$ with values in $\mathbb{R}$ ), but the following can easily be extended to multicriteria problems.

The scope of local sensitivity analysis is to compute exactly and efficiently the sensitivities of the system's response to variations in the system's parameters, around their nominal values.

This is translated by finding

$$
S_{\alpha}=\nabla_{\alpha} J(\alpha)=\left(\frac{\partial J}{\partial \alpha_{1}}(\alpha), \ldots, \frac{\partial J}{\partial \alpha_{p}}(\alpha)\right)^{T},
$$

where $S_{\alpha}$ is the local sensitivity vector of $J$ to variations in $\alpha$. It is a local sensitivity because it depends on the current estimate of $\alpha$ (and of $\boldsymbol{x}_{0}$ ).

Rewriting Eq. (12) as $J(\alpha)=\langle\varphi(\alpha), \varphi(\alpha)\rangle$, where $\langle.,$. defines a dot product on $\mathbb{R}^{m}$. The Gâteaux derivative of $J$ in any direction $\delta \alpha$ reads

$$
\begin{aligned}
\mathrm{d} J(\alpha)[\delta \alpha] & =\left\langle\varphi(\alpha), \frac{\partial \varphi}{\partial \alpha} \cdot \delta \alpha\right\rangle=\left\langle\left[\frac{\partial \varphi}{\partial \alpha}\right]^{*} \cdot \varphi(\alpha), \delta \alpha\right\rangle \\
& \equiv\langle\nabla J, \delta \alpha\rangle .
\end{aligned}
$$

Hence $\left[\frac{\partial \varphi}{\partial \alpha}\right]^{*}$, the adjoint of $\varphi$, allows for the exact computation of the $p$ components of $\nabla J$ at once.

One can find an example of application of such methods with NEMOTAM on the Mercator Ocean's GLORYS 1/4 global ocean reanalysis in Vidard et al. (2011). The initial 
objective was to try to estimate the influences of geographical areas to reduce the forecast error using an adjoint method to compute the sensitivities (while the GLORYS assimilation system is based on optimal interpolation). We conducted a preliminary study by considering the misfit to observations as a proxy of the forecast error and sought to determine the sensitivity of this difference to changes in the initial condition and/or to forcing. That should give an indication about the important phenomena to consider to improve this system.

The most easily interpreted case in this study is to consider a sensitivity criterion coming from the difference in sea surface temperature (SST) maps at the final instant of the assimilation cycle because of its dense coverage in space. This can be translated into computing the gradient:

$J\left(\boldsymbol{x}_{0}, \boldsymbol{q}\right)=\frac{1}{2} \sum_{n=1}^{N_{\mathrm{SST}}}\left\|H_{\mathrm{SST}}\left(\boldsymbol{x}_{n}\right)-\mathrm{SST}^{\mathrm{obs}}\right\|_{\mathbf{R}^{-1}}^{2}$

with a control vector made of $\boldsymbol{x}_{0}=\left(u_{0}, v_{0}, T_{0}, S_{0}, \eta_{0}\right)^{T}$ the initial state vector (current velocities component, temperature, salinity and sea level) and of $\boldsymbol{q}=\left(q_{\mathrm{sr}}, q_{\mathrm{ns}}, \mathrm{emp}\right)^{T}$ (radiative fluxes, total heat fluxes, freshwater fluxes). One can see an example of sensitivity to initial temperature (surface and $100 \mathrm{~m}$ ) as shown in the two bottom panels of Fig. 1. High sensitivity will give a signal similar to the gap in observations (top left), while low sensitivity will show a white area. In this example it is clear that the SST misfit is highly sensitive to changes in surface temperature where the initial mixed layer depth (top right) is low and insensitive elsewhere. The opposite conclusion can be drawn from the sensitivity to the initial temperature at $100 \mathrm{~m}$. This is obviously not a surprise, and it corresponds more to the purpose of verification of the model rather than the original goal of assimilation system improvement. However it highlights the importance of having a good estimate of the vertical mixing and echoes the fact they this vertical mixing is often perturbed by data assimilation. Other components of the gradient show the important role of atmospheric forcing (which again we could have guessed), and ways to improve the system also appear to point to that direction. With the objective of improving the data assimilation system, this approach is obviously not completely satisfactory because, strictly speaking, the assimilation system should be included in the optimality system. In theory, this assimilation system being linear and made of matrix multiplication, deriving its adjoint should be easy. In practice it is a different story - manipulating an operational system is never easy.

The sensitivities are of interest by themselves, but they can also be used for optimising the system. In particular this way of computing gradients is extensively used in variational data assimilation for the minimisation of similar cost function (4D-Var). For ocean application, historically the preferred choice of data assimilation technique has been (and still is for many cases) that of optimal interpolation or 3DVar type schemes. These algorithms make the assumptions that the system state is stationary over a given time window (typically 1 to 10 days), which can be a crude approximation. 4D-Var does not make this assumption and uses the adjoint model to compute the gradient of a cost function of the form

$$
\begin{aligned}
J\left(\boldsymbol{x}_{0}\right) & =\frac{1}{2}\left\|\boldsymbol{x}_{0}-\boldsymbol{x}^{\mathrm{b}}\right\|_{\mathbf{B}^{-1}}^{2} \\
& +\frac{1}{2} \sum_{t=1}^{T}\left\|H_{t}\left(\mathcal{M}\left(\boldsymbol{x}_{0}, t\right)\right)-\boldsymbol{y}_{t}^{\mathrm{obs}}\right\|_{\mathbf{R}^{-1}}^{2},
\end{aligned}
$$

where $\|z\|_{\mathbf{C}}^{2}=\langle z, \mathbf{C} z\rangle$. B is the background and $\mathbf{R}$ the observation error covariance matrix, $\boldsymbol{x}^{\mathrm{b}}$ its the background state, and $\boldsymbol{y}^{\mathrm{obs}}$ are the observations. The gradient $\nabla J$ of this cost function can be computed using relation (14):

$$
\begin{aligned}
\nabla J & =\mathbf{B}^{-1}\left(\boldsymbol{x}_{0}-\boldsymbol{x}^{\mathrm{b}}\right) \\
& +\sum_{t=1}^{T} \mathbf{L}^{*} \mathbf{H}_{t}^{*} \mathbf{R}^{-1}\left(H_{t}\left(\mathcal{M}\left(\boldsymbol{x}_{0}, t\right)\right)-\boldsymbol{y}_{t}^{\mathrm{obs}}\right) .
\end{aligned}
$$

To illustrate the application of 3D-Var and 4D-Var type schemes, one can perform single observation experiments, where only one observation at the end of the assimilation window is assimilated. In that case, after a bit of algebra and assuming $\mathcal{M}\left(\boldsymbol{x}_{0}+\delta \boldsymbol{x}, T\right)=\mathcal{M}\left(\boldsymbol{x}_{0}, T\right)+\mathbf{L} . \delta \boldsymbol{x}$ one can write the optimal state $x^{\text {a }}$ that minimises $J$ as

$$
\begin{aligned}
\boldsymbol{x}^{\mathrm{a}} & =\boldsymbol{x}^{\mathrm{b}} \\
& +\mathbf{B L}^{*} \mathbf{H}^{*}\left(\mathbf{R}+\mathbf{H L B L}^{*} \mathbf{H}^{*}\right)^{-1}\left(H_{T}\left(\mathcal{M}\left(\boldsymbol{x}_{0}, T\right)\right)-\boldsymbol{y}_{T}^{\mathrm{obs}}\right) .
\end{aligned}
$$

For a single observation experiment, it is easy to see that $\left(\mathbf{R}+\mathbf{H L B L}^{*} \mathbf{H}^{*}\right)^{-1}\left(H_{T}\left(\mathcal{M}\left(\boldsymbol{x}_{0}, T\right)\right)-\boldsymbol{y}_{T}^{\text {obs }}\right)$ is a scalar, and when multiplied by $\mathbf{H}^{*}$ it becomes a vector in the state space, with only one non-zero value (assuming the observation is at a grid point). In $3 \mathrm{D}$-Var formulation, $\mathbf{L}^{*}$ is approximated by the identity operator, so the correction to the initial condition outside the observed grid point is solely driven by the prescribed background error statistics in $\mathbf{B}$, while in 4D-Var the model dynamics are accounted for through the adjoint model $\mathbf{L}^{*}$.

An example of such differences is given in Fig. 2 where a single synthetic sea surface height observation, close to the middle of the regional model, at the end of the data assimilation time window, is assimilated using both 3D-Var and incremental 4D-Var algorithms from the NEMOVAR system with NEMO's SEABASS configuration (see Appendix A). The observation misfit value is $0.5 \mathrm{~m}$.

The 3D-Var increment (top figure) shows a perfect Gaussian shape, centred around the observation location, with a maximum amplitude close to the observation value. This Gaussian shape is exactly what is prescribed in the background error covariance matrix $\mathbf{B}$, and the computed increment is independent of the length of the assimilation window. On the other hand, 4D-Var increment is sensitive to the assimilation window length. Two examples are given: the 

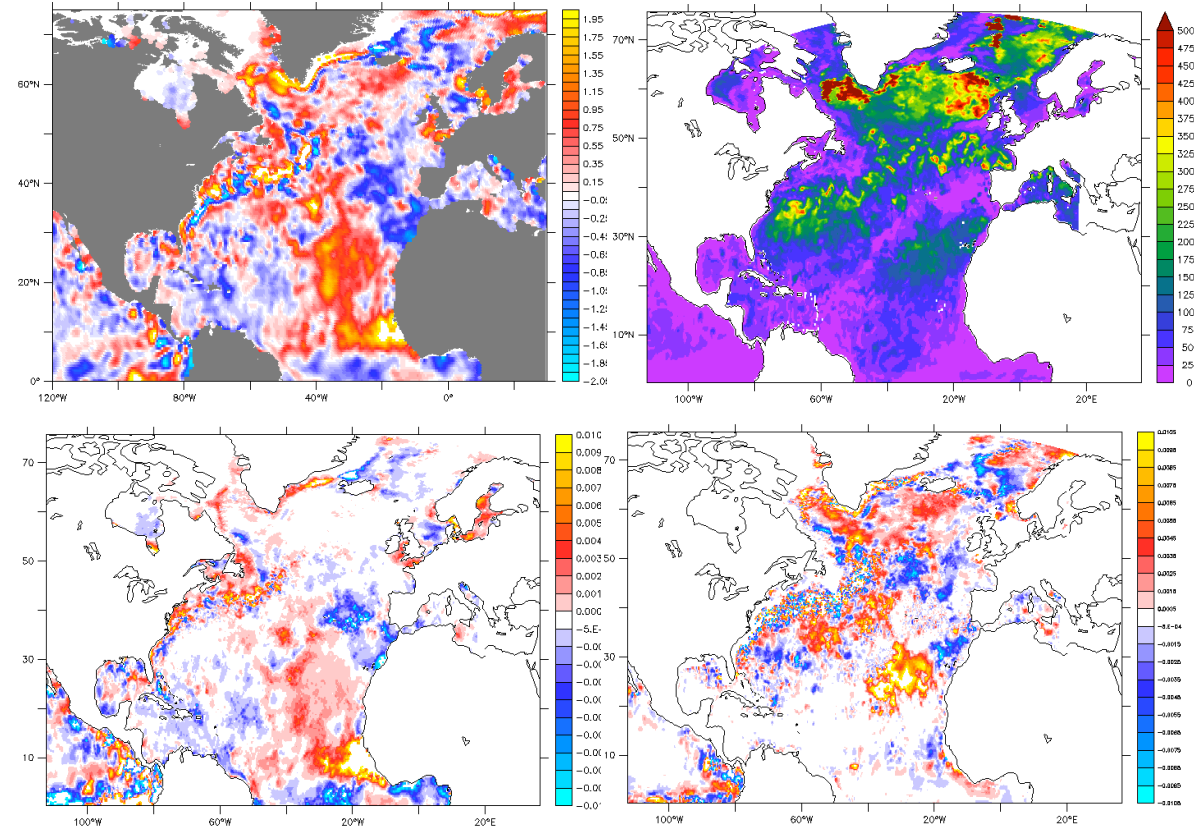

Figure 1. (top) Misfit between forecast and observed SST (left) and mixed layer depth (right). (bottom) Sensitivity to 1-week lead time SST error with respect to variations in initial surface (left) and $100 \mathrm{~m}$ (right) temperature (courtesy of E. Rémy, Mercator Ocean).

first with a 5-day window (bottom left) and the second with a 30-day window. In the first case, the 3D-Var approximation is acceptable, so both 3D-Var and 4D-Var are similar, even though the latter is slightly deformed and displaced to account for the short-term dynamics. For the longer assimilation window (bottom right) however, the effect of the dynamics is more complex; in particular the non-linearities are more developed. As a consequence the 3D-Var approximation is no longer valid, and the shape of the optimal correction is completely different.

This obviously comes at a cost, since 3D-Var would only require one direct model integration and 4D-Var would additionally require one tangent and adjoint integration per minimisation iteration. In a single observation experiment as presented above, the minimisation converges in only one iteration, limiting the cost of 4D-Var to about 4 times that of 3DVar. In a more realistic configuration one performs about 30 to 50 iterations, leading 4D-Var to be up to 200 times more cpu-expensive than 3D-Var. This is why, in many implementations, the minimisation is performed at a lower resolution than the forecast.

\subsection{Singular vectors}

Another application of tangent and adjoint models is stability analysis, which is the study of perturbations on the system. Particular tools for such analysis are the so-called singular vectors.
We classically define the growth rate of a given perturbation $\delta \boldsymbol{x}_{0}$ by

$\rho\left(\delta \boldsymbol{x}_{0}\right)=\frac{\left\|\mathcal{M}\left(\boldsymbol{x}_{0}+\delta \boldsymbol{x}_{0}, T\right)-\mathcal{M}\left(\boldsymbol{x}_{0}, T\right)\right\|_{1}}{\left\|\delta \boldsymbol{x}_{0}\right\|_{2}}$,

where $\|.\|_{1}=\left\langle., \mathbf{W}_{1}.\right\rangle$ and $\|.\|_{2}=\left\langle., \mathbf{W}_{2}.\right\rangle$ are given norms and $T$ is the final time of the considered window.

One can then define the optimal perturbation $\delta x_{0}^{1}$ so that $\rho\left(\delta \boldsymbol{x}_{0}^{1}\right)=\max _{\delta \boldsymbol{x}_{0}} \rho\left(\delta \boldsymbol{x}_{0}\right)$ and then deduce a family of maximum growth vectors

$\rho\left(\delta \boldsymbol{x}_{0}^{i}\right)=\max _{\delta \boldsymbol{x}_{0} \perp \operatorname{Span}\left(\delta \boldsymbol{x}_{0}^{1}, \ldots, \delta \boldsymbol{x}_{0}^{i-1}\right)} \rho\left(\delta \boldsymbol{x}_{0}\right), \quad i \geq 2$.

By restricting the study to the linear part of the perturbation behaviour, the growth rate becomes (denoting $\mathbf{L}=$ $\mathbf{L}(\boldsymbol{x}, T)$ for clarity)

$$
\begin{aligned}
\rho^{2}\left(\delta \boldsymbol{x}_{0}\right)=\frac{\left\|\mathbf{L} \delta \boldsymbol{x}_{0}\right\|_{1}^{2}}{\left\|\delta \boldsymbol{x}_{0}\right\|_{2}^{2}} & =\frac{\left\langle\mathbf{L} \delta \boldsymbol{x}_{0}, \mathbf{W}_{1} \mathbf{L} \delta \boldsymbol{x}_{0}\right\rangle}{\left\langle\delta \boldsymbol{x}_{0}, \mathbf{W}_{2} \delta \boldsymbol{x}_{0}\right\rangle} \\
& =\frac{\left\langle\delta \boldsymbol{x}_{0}, \mathbf{L}^{*} \mathbf{W}_{1} \mathbf{L} \delta \boldsymbol{x}_{0}\right\rangle}{\left\langle\delta \boldsymbol{x}_{0}, \mathbf{W}_{2} \delta \boldsymbol{x}_{0}\right\rangle} .
\end{aligned}
$$

Maximising the above equation is equivalent to solving the following generalised eigenvalue problem:

$\mathbf{L}^{*} \mathbf{W}_{1} \mathbf{L} g_{i}^{+}=\mu_{i} \mathbf{W}_{2} g_{i}^{+}$, 


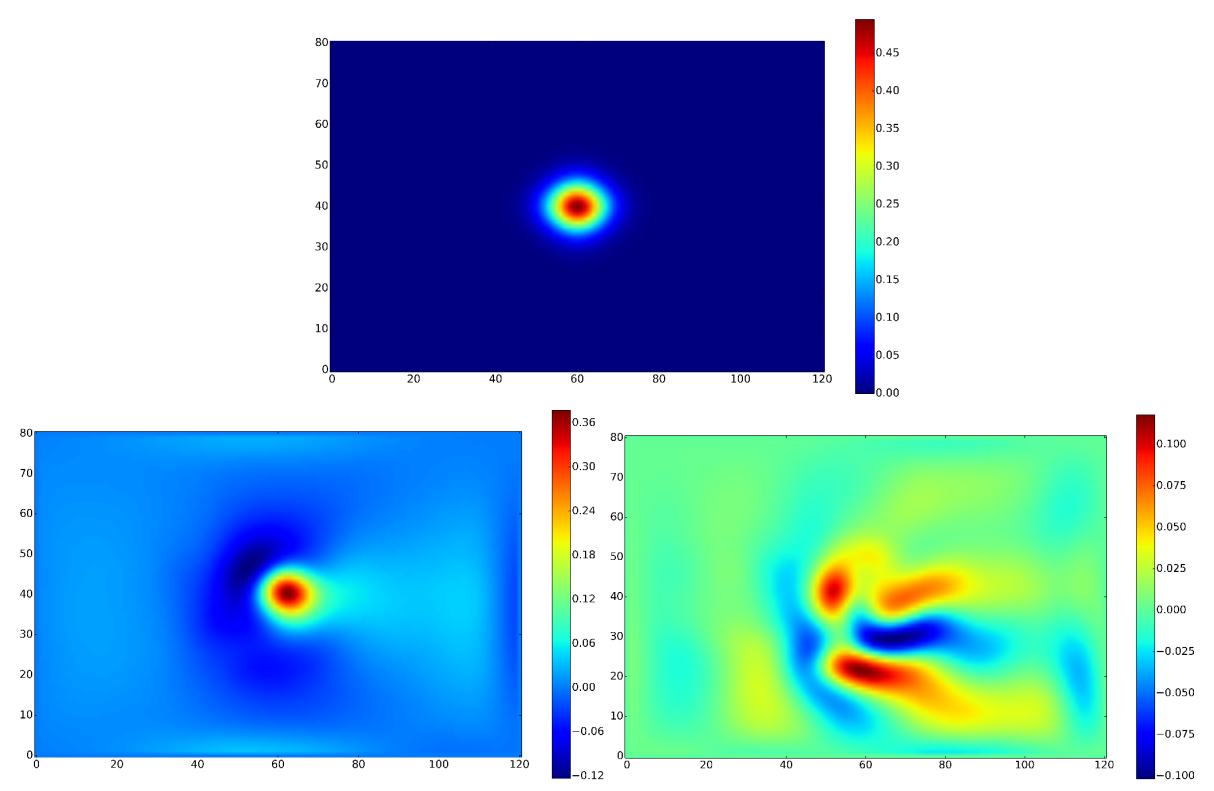

Figure 2. Assimilation increments in the SEABASS configuration corresponding to one sea surface height observation and a misfit of $0.5 \mathrm{~m}$. Coming from NEMOVAR 3D-Var (top) and 4D-Var (bottom) formulation. Two different assimilation window lengths are presented for the 4D-Var: 5 days (left) and 30 days (right).

which is equivalent, using the change of variable $g_{i}^{+}=$ $\mathbf{W}_{2}^{-1 / 2} f_{i}^{+}$, to

$\mathbf{W}_{2}^{-1 / 2} \mathbf{L}^{*} \mathbf{W}_{1} \mathbf{L} \mathbf{W}_{2}^{-1 / 2} f_{i}^{+}=\mu_{i} f_{i}^{+}$.

$\mathbf{W}_{2}^{-1 / 2} \mathbf{L}^{*} \mathbf{W}_{1} \mathbf{L} \mathbf{W}_{2}^{-1 / 2}$ being a symmetric positive definite matrix, its eigenvalues are positive real and its eigenvectors are (or can be chosen) orthonormal. The strongest growth vectors are the eigenvectors of $\mathbf{W}_{2}^{-1 / 2} \mathbf{L}^{*} \mathbf{W}_{1} \mathbf{L} \mathbf{W}_{2}^{-1 / 2}$ corresponding to the greater eigenvalues. They are called forward singular vectors.

The backward singular vectors, denoted $f_{i}^{-}$, are denoted by $\mathbf{W}_{1}^{1 / 2} \mathbf{L} \mathbf{W}_{2}^{-1 / 2} f_{i}^{+}=\sqrt{\mu_{i}} f_{i}^{-}$. The eigenvalue corresponding to $f_{i}^{-}$is $\mu_{i}$ as well. Forward singular vectors represent the directions of perturbation that will grow fastest, while backward singular vector represent the directions of perturbation that have grown the most. $\sqrt{\mu_{i}}$ is the amplification factor associated with the $i$ th singular vector.

The computation of the $f_{i}^{+}$and $f_{i}^{-}$generally requires numerous matrix-vector multiplications, i.e. direct integrations of the model and backward adjoint integrations. The result of these calculations depends on the norm used, the time window and the initial state if the model is non-linear. Examples of such vectors for a 1/12th of degree SEABASS configuration are shown in Fig. 3. The left panel shows the sea surface height component of the first forward singular vector that exhibits a strong signal over the dominant jet of this configuration, showing that the optimal perturbation is located in the most active region (as it is shown in Fig. A1). The right panel shows the corresponding backward singular vector, i.e. the result of the evolution of the optimal perturbation through the linear part of the dynamics. The complex structure of the original perturbation has been transformed into several individual vortices. This is similar to what Durbiano (2001) presented for a shallow-water model.

These singular vectors were computed using an energy norm to define $\|.\|_{1,2}$ and the parpack (Lehoucq et al., 1998) external library to perform the singular value decomposition. The computational cost required for obtaining singular vectors can vary from one situation to another since it depends on the number of iterations the Arnoldi algorithm used in parpack takes to converge, which itself depends on the eigenspectrum. For instance it took from 27 to 49 iterations for computing the 10 leading singular vectors of the different cases discussed in Fig. 4, each iterations requiring the integration of both tangent and adjoint models.

These vectors, thanks to the information they contain about the system behaviour, have many applications. Among them, one can cite ensemble forecast, sensitivity studies (Rivière et al., 2009, for a recent application), the order reduction in data assimilation (Blayo et al., 2003), improving the monitoring network (Qin and $\mathrm{Mu}, 2011$ ) or allowing one to better select targeted observations (Mu et al., 2009).

A by-product of the computation of singular vectors is the amplification factor $\sqrt{\mu_{i}}$ that represents the growth of the corresponding singular vector at $T$ the end of the time window. Figure 4 shows the impact of the length of this time window (left) and of the model resolution (right). The impact of the latter is obvious: the higher the resolution the more active the model - hence the faster perturbations amplify. Regard- 

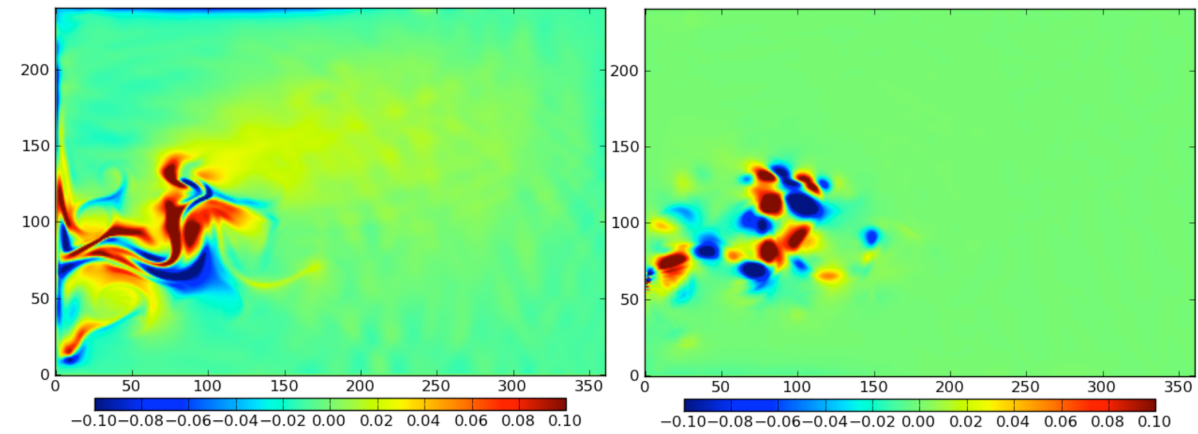

Figure 3. Sea surface height component of the leading forward (left) and backward (right) singular vectors for a SEABASS configuration at $1 / 12^{\circ}$ and a 10-day window.
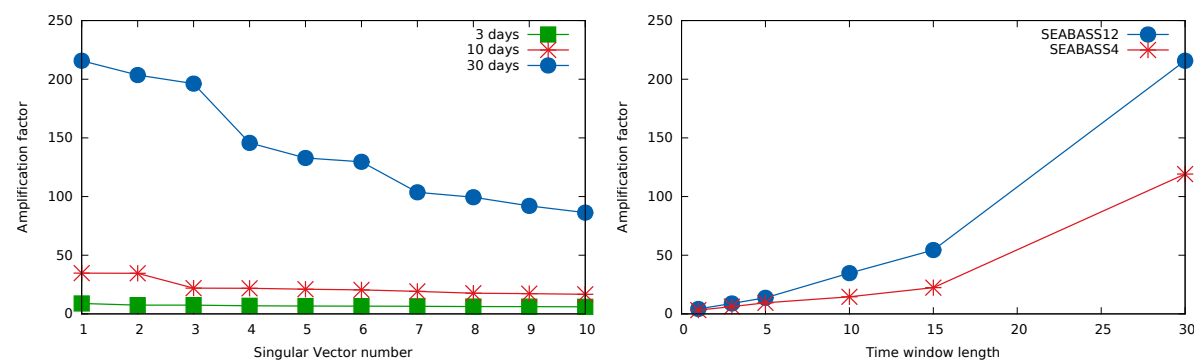

Figure 4. Amplification factor for the 10 first singular vectors of the $1 / 12^{\circ}$ SEABASS configuration for several time windows (left) and comparison of the amplification factors of the first singular vector for the $1 / 12^{\circ}$ and the $1 / 4^{\circ}$ SEABASS configurations (right).

ing the time window length it is less obvious. For a short period of time as presented here the link is the same. Perturbations get more time to grow; therefore, the amplification factor increases. With a longer time window (few years or decades) optimal perturbations are such that amplification factors will start to decrease. This dipping point is important in predictability studies (see Zanna et al., 2011, for more details on this topic).

\section{Conclusions}

The tangent and adjoint models of NEMO (NEMOTAM) are now available (with respect to the 3.4.1 version of NEMO at the time of writing this paper). It is part of the NEMOASSIM tools (Bouttier et al., 2012), whose aim is to ease the interface between NEMO code and most data assimilation algorithms. In the few preceding pages, these models, the technical choices made for their developments and their validation were discussed. Additionally some applications were presented as an illustration of potential use.

When developing a TAM, two main difficulties have to be addressed: the handling of the non-linearities and of the nondifferentiable parts. Indeed non-linear equations require the storage and/or recomputation of the non-linear trajectory to differentiate around. In NEMOTAM this is done through the so-called checkpointing strategy, consisting in saving part of the non-linear trajectory at a given frequency (checkpoints), recomputing the missing part and, if needed, interpolating it linearly between checkpoints. On the other hand the nondifferentiability issues are dealt with using three different approaches, depending on the discontinuity nature: numerical or physical regularisation, numerical or physical approximation and non-linear branching.

All these choices have to be validated along with the coding itself. To that end a significant effort has been invested in NEMOTAM. First, adjoint tests are systematically implemented for each adjoint routine and give an exact indication of the validity of the adjoint code. For the tangent linear model, there is no exact test which can strictly validate the development. However, comparing the propagation of a small perturbation by the tangent-linear model and the direct model gives an idea of the validity of the tangent-linear hypothesis. Finally, an estimator of the errors due to the approximations of NEMO non-differentiable parts is also provided for the corresponding routines.

The range of applications using NEMOTAM is wide. To illustrate that, three example applications were presented. First a local sensitivity analysis with a realistic NEMO configuration was carried out. Then, a very simple data assimilation experiment, using a single observation is also performed, illustrating the impact of the use of an adjoint model. And finally some singular vectors were computed using NEMOTAM. 
The scope of the current NEMOTAM implementation leaves room for different extensions (e.g. taking in account other NEMO modules, such as the sea ice model, nested grids). In order to handle properly the multi-processor aspects and optimise computing cost, the current NEMOTAM is hand-coded. Such an approach can limit the range of possible input quantities to be considered when computing sensitivities. As it is, it can compute - without any change of code - sensitivities to perturbations to the initial condition and/or surface boundary conditions, and with very limited modifications, sensitivities to perturbation of physical parameters (such as bottom friction for instance). However for parameters that are used throughout the model code, such as gridrelated parameters, it would require a significant amount of coding. This is why, in order to provide more flexibility in the choice of variable to differentiate around and to ease the process of updating the NEMOTAM code with new features and following the evolution of the direct code, a subtle tradeoff between automatic differentiation and manual intervention could be very beneficial. 


\section{Appendix A: SEABASS configuration}

The SEABASS reference configuration for NEMO is mentioned several times in this paper. This configuration is an academic basin presenting a double-gyre circulation. The horizontal domain extends from 24 to $44^{\circ} \mathrm{N}$ and over $30^{\circ}$ in longitude. For a $1 / 4^{\circ}$ horizontal resolution, the grid contains only 121 points in longitude and 81 points in latitude. The time step is $900 \mathrm{~s}$. The ocean is sliced into 11 verticals levels, from surface to $4000 \mathrm{~m}$, described with a $z$ coordinate. The domain is closed and has a flat bottom. Lateral boundaries conditions are frictionless and bottom boundary condition exerts a linear friction. The circulation is only forced by a zonal wind. Lateral dissipation is performed on dynamics and tracers with a biharmonic diffusion operator. The salinity is constant over the whole domain, and the initial stratification is produced using an analytical temperature profile. Details can be found in Cosme et al. (2010), for example.

Even if SEABASS is an academic configuration, it exhibits a turbulence level statistically meaningful regarding the eddy activity and the non-linearity amplitude of the actual Gulf Stream system. This SEABASS characteristic is interesting in a data assimilation context, as the oceanic turbulence is one of the major current stakes for data assimilation methods in oceanography.

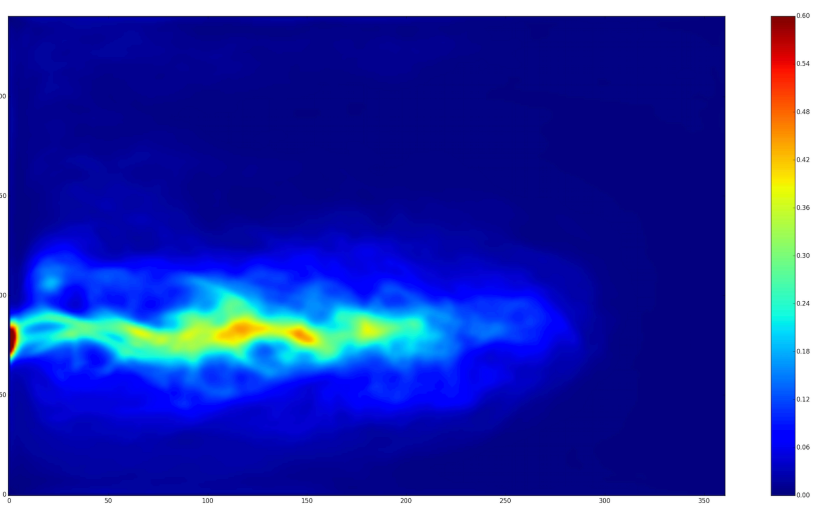

Figure A1. Surface eddy kinetic energy averaged over 1 year (in $\mathrm{m}^{2} \mathrm{~s}^{-2}$ ), for the SEABASS configuration at a $1 / 12^{\circ}$ horizontal resolution. 


\section{Code availability}

NEMOTAM was released with NEMO 3.4 STABLE version for the first time as an official NEMO component. To extract this version of NEMO, users need to create an account on the NEMO website (www.nemo-ocean.eu/user/register). After that, source code can be utilised by using the relevant SVN command. Instructions to compile and to run NEMOTAM can be found at this URL (visible once logged in): http://www.nemo-ocean.eu/Using-NEMO/User-Guides/ Basics/Tangent-and-Adjoint-quick-start-guide.

Acknowledgements. The authors would like to thanks the NEMOVAR group in general and A. Weaver and K. Mogensen in particular for their participation in the early phase of the development of NEMOTAM. Additional precious help came from R. Benshila, who guided us through the meander of the NEMO system. This work was also financially supported by CNES and the French National Research Agency (ANR project VODA: ANR-08-COSI-016).

Edited by: D. Ham

\section{References}

Blayo, E., Durbiano, S., Vidard, A., and Le Dimet, F.-X.: Reduced order strategies for variational data assimilation in oceanic models, in: Data Assimilation for Geophysical Flows, edited by: Sportisse, B. and Le Dimet, F.-X., 18 pp., Springer-Verlag, 2003.

Bouttier, P.-A., Blayo, E., Brankart, J.-M., Brasseur, P., Cosme, E., Verron, J., and Vidard, A.: Toward a data assimilation system for NEMO, Mercator Ocean Quarterly Newsletter, 46, 24-30, 2012.

Daget, N., Weaver, A. T., and Balmaseda, M. A.: Ensemble estimation of background-error variances in a three-dimensional variational data assimilation system for the global ocean, Q. J. Roy. Meteor. Soc., 135, 1071-1094, 2009.

Deltel, C.: Estimation de la circulation dans l'océan Atlantique Sud par assimilation variationnelle de données in situ, Impact du contrôle optimal des forçages et de l'hydrologie aux frontières ouvertes, 185 pp., PhD thesis, Univ. de Bretagne Occidentale, 2002.

Durbiano, S.: Vecteurs caractéristiques de modèles océaniques pour la réduction d'ordre en assimilation de données, $\mathrm{PhD}$ thesis, Université Joseph Fourier, 2001.

Errico, R. M.: What Is an Adjoint Model?, B. Am. Meteorol. Soc., 78, 2577-2591, 1997.

Forget, G., Ferron, B., and Mercier, H.: Combining Argo profiles with a general circulation model in the North Atlantic. Part 1: Estimation of hydrographic and circulation anomalies from synthetic profiles, over a year, Ocean Model., 20, 1-16, 2008.

Giering, R. and Kaminski, T.: Recipes for adjoint code construction, ACM Trans. Math. Softw., 24, 437-474, 1998.

Hascoët, L. and Pascual, V.: TAPENADE 2.1 user's guide, Technical Report 0300, INRIA, 2004.

Lawless, A., Nichols, N., and Ballard, S.: A comparison of two methods for developing the linearization of a shallow-water model, Q. J. Roy. Meteor. Soc., 129, 1237-1254, 2003.
Lehoucq, R. B., Sorensen, D. C., and Yang, C.: ARPACK Users' Guide: Solution of Large-scale Eigenvalue Problems with Implicitly Restarted Arnoldi Methods, SIAM Philadelphia, ISBN 0898714079, 142 pp., 1998.

Madec, G.: NEMO ocean engine, Note du Pôle de modélisation, Institut Pierre-Simon Laplace (IPSL), France, No 27, ISSN No 1288-1619, 2008.

Madec, G., Delecluse, P., Imbard, M., and Lévy, C.: OpA 8.1 ocean general circulation model reference manual, IPSL, Note No. 11 du Pôle de Modélisation (LODYC), 1998.

Marotzke, J., Giering, R., Zhang, K. Q., Stammer, D., Hill, C., and Lee, T.: Construction of the adjoint MIT ocean general circulation model and application to Atlantic heat transport sensitivity, J. Geophys. Res., 104, 29529-29547, 1999.

Mogensen, K. S., Balmaseda, M. A., Anthony, W., Martin, M., and Vidard, A.: NEMOVAR: A variational data assimilation system for the NEMO ocean model, ECMWF newsletter, 120, 17-21, 2009.

Moore, A., Vialard, J., Weaver, A., Anderson, D., Kleeman, R., and Johnson, J.: The Role of Air-Sea Interaction in Controlling the Optimal Perturbations of Low-Frequency Tropical Coupled Ocean-Atmosphere Modes, J. Climate, 16, 951-968, 2003.

Moore, A., Arango, H., Di Lorenzo, E., Cornuelle, B., Miller, A., and Neilson, D.: A comprehensive ocean prediction and analysis system based on the tangent linear and adjoint of a regional ocean model, Ocean Model., 7, 227-258, 2004.

Mu, M., Zhou, F., and Wang, H.: A Method for Identifying the Sensitive Areas in Targeted Observations for Tropical Cyclone Prediction: Conditional Nonlinear Optimal Perturbation, Mon. Weather Rev., 137, 1623-1639, 2009.

Qin, X. and Mu, M.: Influence of conditional nonlinear optimal perturbations sensitivity on typhoon track forecasts, Q. J. Roy. Meteor. Soc., 138, 185-197, 2011.

Rémy, E.: Assimilation variationnelle de données tomographiques simulées dans des modèles de circulation océanique, $\mathrm{PhD}$ thesis, Univ. Paris 6, 1999.

Rivière, O., Lapeyre, G., and Talagrand, O.: A novel technique for nonlinear sensitivity analysis: application to moist predictability, Q. J. Roy. Meteor. Soc., 135, 1520-1537, 2009.

Sévellec, F., Huck, T., BenJelloul, M., Grima, N., Vialard, J., and Weaver, A.: Optimal surface salinity perturbations of the meridional overturning and heat transport in a global ocean general circulation model, J. Phys. Oceanogr., 38, 2739-2754, 2008.

Sévellec, F., Huck, T., BenJelloul, M., and Vialard, J.: Non-normal multidecadal response of the thermohaline circulation induced by optimal surface salinity perturbations, J. Phys. Oceanogr., 39, 852-872, 2009.

Tber, M. H., Hascoet, L., Vidard, A., and Dauvergne, B.: Building the Tangent and Adjoint codes of the Ocean General Circulation Model OPA with the Automatic Differentiation tool TAPENADE, Research Report RR-6372, INRIA, 2007.

Thuburn, J.: Adjoints of Nonoscillatory Advection Schemes, J. Comput. Phys., 171, 616-631, 2001.

Vidard, A.: Vers une prise en compte de l'erreur modèle en assimilation de données 4D-variationnelle, $\mathrm{PhD}$ thesis, Université Joseph Fourier, 2001.

Vidard, A., Rémy, E., and Greiner, E.: Sensitivity analysis through adjoint method: application to the GLORYS reanalysis, Contrat no. 08/D43, Mercator Océan, 2011. 
Vossepoel, F. C., Weaver, A., Vialard, J., and Delecluse, P.: Adjustment of near-equatorial wind stress with 4D-Var data assimilation in a model of the Pacific Ocean, Mon. Weather Rev., 132, 2070-2083, 2003.

Weaver, A. T., Vialard, J., and Anderson, D. L. T.: Three- and FourDimensional Variational Assimilation with a General Circulation Model of the Tropical Pacific Ocean. Part I: Formulation, Internal Diagnostics, and Consistency Checks, Mon. Weather Rev., 131, 1360-1378, 2003.
Weaver, A. T., Deltel, C., Machu, E., and Ricci, S.: A multivariate balance operator for variational ocean data assimilation, Q. J. Roy. Meteor. Soc., 131, 3605-3625, 2005.

Zanna, L., Heimbach, P., Moore, A. M., and Tziperman, E.: Upperocean singular vectors of the North Atlantic climate with implications for linear predictability and variability, Q. J. Roy. Meteor. Soc., 138, 500-513, 2011. 